\title{
STATISTICAL MECHANICS AS GUIDANCE FOR PARTICLE-BASED COMPUTATIONAL METHODS
}

\author{
IGNACIO G. TEJADA* AND RAFAEL JIMENEZ ${ }^{\dagger}$ \\ *Dpto. Ingeniería y Morfología del Terreno \\ Universidad Politécnica de Madrid \\ C/ Profesor Aranguren, s/n 28040 Madrid (Spain) \\ e-mail: igtejada@caminos.upm.es/ \\ $\dagger$ ditto. \\ e-mail: rjimenez@caminos.upm.es
}

Key words: Granular Materials, Statistical Mechanics

\begin{abstract}
Particle-based methods apply some laws of Classical Mechanics to all the particles of a granular system. On the other hand, classical Statistical Mechanics deals with systems consisting of a lot of particles, focusing on the statistical distribution of some intensive properties. Consequently, the macroscopic behaviour and the average properties of the system in equilibrium are based on some microscopic considerations. To do that, statistical tools and mechanical laws are used together.

For an implemented particle-based method to be realistic enough, then the obtained simulations should satisfy some basic underlying physical requirements, and Statistical Mechanics is a useful tool to establish such requirements.

This paper presents some results based on Statistical Mechanics that are useful for a realistic modelling of granular systems using Particle-based methods.

Examples of the discussed issues include that the same protocol makes a disordered granular medium attain always the same packing ratio; that some local arrangements are less probable than others; that there is a well-established limit to the achievable density of granular assemblies of hard spheres; and that the equilibrium of disordered granular systems does not coincide to the state of minimum potential energy (which would be get in a completely ordered arrangement).

The consideration of such issues in practical applications could be helpful to save time in computational methods, to avoid mistakes or, at least, to verify that the implementations are realistic.
\end{abstract}




\section{INTRODUCTION}

Engineers are usually concerned about finding which are the stress-strain relationships of granular materials. The complex nature of these media does not make it possible to propose an universal law, or to establish a simple relationship which included all the observed features. In fact, relationships use to be not linear and use to change during static, dynamic and cyclic modifications of the stresses. In consequence many constitutive models have been developed to obtain the stress-strain relationships for jammed granular media by comparing them to special continuum media (macromechanics) [1, 2].

Many of the constitutive equations consider the stress-strain history of the material, which will be decisive for the future behavior. Although today is still a pipe dream finding a universal constitutive law, some results are starting to do it possible for the simplest media. If there was a reproducible state of equilibrium of every medium, it would be possible to propose an equation of state, rather than a constitutive equation [3, 4]. An equation of state is a unique function which relates all the intervening properties of the medium in the equilibrium state. It could be:

$f$ (size distribution, shape, constitutive properties, roughness, arrangement $)=0$

On the other hand, and since the difficulty lies mostly on the complex particular nature of these media, some models focus on the main features of the particles (micromechanics). In particular, since the DEM was proposed by Cundall and Strack [5], particle-based numerical models are being developed. These methods are usually based on the arrangement of virtual individual particles (using simple constitutive equations and solving numerically such equations for the whole interacting system). Saving computational time and ensuring realistic simulations are the aim of a lot of research in this area.

A complement for numerical particle-based methods could be particle-based theoretical approaches. By applying several physical principles, these approaches could be useful to understand some basic properties of granular media. In this paper, an introduction to approaches based on Statistical Mechanics is presented. They are compared to other techniques based on classical mechanics (such as DEM, molecular dynamics, etc.) in Figure 1. Some fundamentals on these techniques are summarized in sections below.

\section{FUNDAMENTALS OF STATISTICAL MECHANICS}

\subsection{WHY USING STATISTICAL MECHANICS?}

Thermodynamics studies the conditions of equilibrium and the laws governing the exchanges of various forms of energy or matter of a system which interacts with its surrounding. These laws or principles have been traditionally developed for thermal systems, but they can be applied to other systems, such as granular media. 


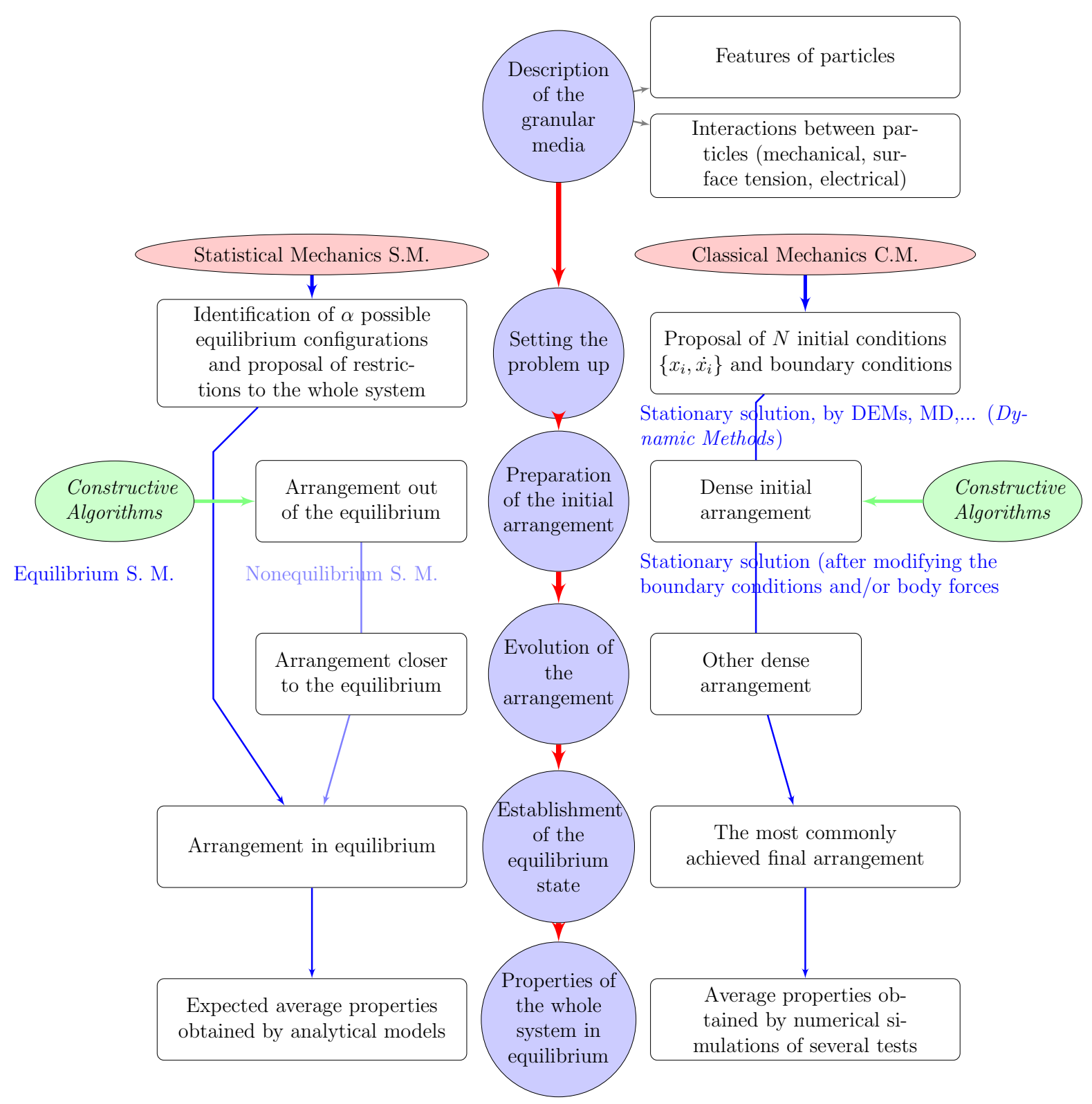

Figure 1: Comparison between statistical mechanics and classical mechanics approaches of particle-based methods 
Statistical mechanics (SM) [6, 7] deals with systems consisting of a lot of particles, describing the macroscopic behavior of systems using microscopic considerations. To do that, statistical tools and mechanical laws are used together. Because granular media consist of a a lot of interacting particles, applying these techniques could be interesting.

\subsection{DEFINITIONS AND BASIC HYPOTHESIS}

Traditionally, statistical mechanics considers physical systems composed of $N$ identical particles confined to a space of volume $V$. Thermodynamic limit conditions means: $N \rightarrow \infty, V \rightarrow \infty$ and $\frac{N}{V}=C$. For granular media, this condition can be probably achieved just imposing that $N \rightarrow \infty$ and that the boundary conditions effects are disregarded $^{1}$. In this limit, the extensive properties are proportional to the size of the system, $\mathrm{N}$, while the intensive properties become independent thereof.

A macrostate of the system is defined according to some macroscopic properties (the state variables), so that if the properties do not change, the macrostate does not change. For instance, the macrostate of a jammed granular system could be described by the number of identical particles $N$ and the elastic potential energy $E$ (which depends on the stresses).

A microstate is a microscopic configuration compatible with a particular macrostate. (For instance, every arrangement of $N$ particles in equilibrium with the stress state of a macrostate).

A given macrostate in general corresponds to a large number of microstates and it is supposed that the system in equilibrium ${ }^{2}$ is equally likely to be in any one of these microstates (postulate of equal a priori probabilities). The actual number of possible microstates is expressed as $\Omega_{(N, E)}$.

It is also introduced the ergodic hypothesis: time averages of a system, exploring its mechanically stable states subject to some external drive (e.g., tapping, cyclic stress processes), coincide with suitable ensemble averages over its jammed states. Therefore observing the evolution of a granular system over a driving process for a long time is equivalent to sampling many independent realizations of the same process. The collection of this "mental copies" of the given system is called ensemble. Nevertheless, while for some systems the exploration is produced as time goes by (thermal systems), for dissipative systems (as granular media) energy has to be injected during the driving process.

The phase space $\Gamma$ is the most appropriate workshop for studying the ensembles, by locating the $N$ particles, so that once a particle is placed, its energy is known. The coordinates

\footnotetext{
${ }^{1}$ For media in which there are particles of different sizes, the thermodynamic limit condition means that, if they are put into groups according to their size $\left\{N_{a}, N_{b}, \cdots\right\}$, then $N_{i} \rightarrow \infty$ and $N_{i} / N_{j}=$ Constant.

${ }^{2}$ Note that every microstate (e. g. every arrangement of a granular medium) is in mechanical equilibrium, but this definition of (thermodynamical) equilibrium relates to the statistical distribution of some intensive properties.
} 
of this space $\left(q_{i}, p_{i}\right)$ depend on the nature of the intervening energies, while the dimension depends on the number of particles and on the number of degrees of freedom. For instance, to study the motion of $N$ rigid bodies, the definition of a microstate would require the specification of $3 N$ position coordinates and $3 N$ momentum coordinates, so that the phase space is $6 N$-dimensional.

The object of Statistical Mechanics is to provide a link between expected macroscopic quantities $<B_{(\bar{x}, t)}>$ (which are fields in the physical space-time) and microscopic quantities $b_{\left(q_{i}, p_{i} ; \bar{x}, t\right)}$ (which are functions of the phase-space coordinates). This is achieved via the phase-space distribution function $F_{\left(q_{i}, p_{i}, \bar{x}, t\right)}$ in the way:

$$
<B_{(\bar{x}, t)}>=\int_{\Gamma} b_{\left(q_{i}, p_{i} ; \bar{x}, t\right)} F_{\left(q_{i}, p_{i}, \bar{x}, t\right)} \mathrm{d} q_{i} \mathrm{~d} p_{i}
$$

\subsection{MAXWELL-BOLTZMANN STATISTICS}

Different macroscopic environmental constraints lead to different types of ensembles, with particular statistical characteristics. Within the ensembles considered by SM(see [6]), the canonical ensemble is adequate for systems which interact with it surroundings, exchanging energy. Under this consideration, the systems are in equilibrium with an external "reservoir", and the statistical distribution is governed by a directly observable and controllable parameter (e.g. the temperature for thermal systems). Therefore it is assumed that not all the particles are located at the same energy level, but the distribution is governed by the control parameter of the reservoir.

If the system is non-interacting, then the total energy is given by $E=\sum_{1}^{N} E_{i}$. If there are $r$ energy levels and the number of particles which are placed there are $N_{i}$ (with $1 \leq i \leq r$ ) , then:

$$
N=\sum_{1}^{r} N_{i} \quad E=\sum_{1}^{r} N_{i} E_{i}
$$

There are a lot of possible multisets $\left\{N_{i}\right\}$ by means of which the total energy is the same. However, the number of microstates is given by (permutation of a multiset):

$$
\Omega_{(N, E)}=\frac{N !}{N_{1} ! N_{2} ! \cdots N_{r} !}
$$

According to the postulate of equilibrium, the distribution is obtained by maximizing the equation (3) with the restrictions (2). This can be achieved using Lagrange multipliers and the result is the Maxwell-Boltzmann Statistics [7, 6]:

$$
P_{i}=\frac{N_{i}}{N}=\frac{e^{-E_{i} \beta}}{\sum_{i} e^{-E_{i} \beta}},
$$

As a result, the distribution depends on $\beta$, which relates to the control parameter of the surrounding of the system (e.g. the temperature for thermal systems). 
Accordingly, it can be demonstrated that for systems of $N$ particles with $S_{N}$ degrees of freedom, obeying the classical mechanics laws (the Hamiltonian of the whole system is given by $H_{N\left(q_{i}, p_{i}, \alpha\right)}$, including the external parameters $\alpha$ which have influence on it), a stationary solution which can be reached in equilibrium (corresponding to canonical ensembles) is given by this distribution function:

$$
F_{\left(q_{i}, p_{i}\right)}=Z_{N(\beta, \alpha)}^{-1} e^{-H_{N\left(q_{i}, p_{i}, \alpha\right)} \beta}
$$

where $Z_{N(\beta, \alpha)}$ is called the partition function of the $N$ particles and it is given by

$$
Z_{N(\beta, \alpha)}=\int_{\Gamma} e^{-H_{N\left(p_{i}, q_{i}, \alpha\right)} \beta} \mathrm{d} p_{i} \mathrm{~d} q_{i}
$$

Using (1), the expected value of a variable $B_{(q, p)}$ at equilibrium is given by:

$$
<B_{(q, p)}>=\frac{\iint_{\Gamma} b_{\left(q_{i}, p_{i}\right)} e^{-H_{N\left(p_{i}, q_{i}\right)} \beta} \mathrm{d} q_{i} \mathrm{~d} p_{i}}{\iint_{\Gamma} e^{\left.-H_{N\left(p_{i}, q_{i}\right)}\right)^{\beta}} \mathrm{d} p_{i} \mathrm{~d} q_{i}}
$$

For non-interacting systems, i.e. those systems in which the Hamiltonian is separable $H_{N\left(q_{i}, p_{i}\right)}=\sum_{i=1}^{N} H_{1}\left(q_{i}, p_{i}\right)$ (so that the total energy is the sum of the individual energies of the particles), it is possible to evaluate the expected value by considering the distribution function of the reduced phase space of just one particle $\Gamma^{\prime}$, i.e. expression (7) becomes:

$$
<B_{(q, p)}>=\frac{\iint_{\Gamma^{\prime}} b_{(q, p)} e^{-H_{1(p, q)} \beta} \mathrm{d} q \mathrm{~d} p}{\iint_{\Gamma^{\prime}} e^{-H_{1(p, q)} \beta} \mathrm{d} q \mathrm{~d} p}
$$

Being $H_{1(q, p)}$ the Hamiltonian of just one particle.

\section{APPLICATION OF STATISTICAL MECHANICS TO GRANULAR ME- DIA}

\subsection{BASIC ASSUMPTIONS}

Jammed granular media can be considered as sets of a lot of identical particles interacting via classical forces. The energy exchange with the surroundings is made via the boundary conditions of the elastic problem: external pressures or rigid or deformable walls of the container. As $N$ is very large, the thermodynamic limit condition is assumed. The simplest case is a system consisting of monodisperse (just one diameter size), elastic, cohesionless, frictionless and spherical (3D) or circular (2D) particles.

Just a few frameworks based on statistical mechanics have been developed for describing which is the equilibrium state of a granular medium. Two of them are summarized below. They are focused on defining which coordinates make up the phase space and which is the control parameter $\beta$ (it determines the state of the system). 


\subsection{EDWARDS' APPROACH}

Edwards was the first to propose that a statistical mechanics approach might be feasible to describe dense granular media [8]. A new framework was set up to describe the state of powders. Assuming that granular systems have entropy, it was claimed that the volume plays the role of energy.

As a result, a thermodynamic formalism was established based on a phase space which considers the relative position of the particles, a volume functional which takes the place of the Hamiltonian, the effective volume, Y, which is equivalent to the Helmholtz free energy, and a new variable, the frothiness, $\mathrm{X}$, analogous to temperature. This formalism also introduced the hypothesis that time averages of a system, exploring its mechanically stable states subject to some external drive (e.g., tapping), coincide with suitable ensemble averages over its jammed states.

\subsection{A NEW APPROACH}

Edwards' theory builds on the idea that particle interactions are produced via hardcore potentials and, as kinetic energy is not taken into consideration, only gravitational energy is (implicitly) considered.

A new proposal [9] is based on the idea that the internal stress field (which has to be compatible to the external stress field or the boundary conditions) can be divided in local stress domains. The particles within this stress domains can arrange in several equilibrium configurations and, according to the elastic potential energy, the system is supposed to behave as a canonical ensemble.

In consequence, the energy of a particle of radius $R$ and (elastic) constitutive parameters $C_{i j k l}$, arranged according to a configuration $\alpha$ in equilibrium with a stress field $\sigma_{i j}$ can be expressed as:

$$
e \simeq \frac{1}{2} \sum_{I=1}^{N_{\alpha}} \frac{F_{I, \alpha\left(R, \sigma_{i j}\right)}^{2}}{K_{k, \alpha\left(R, C_{i j k l}\right)}}=H_{1, \alpha\left(R, \sigma_{i j}\right)}
$$

where $N_{\alpha}$ is the number of coordination of the configuration, $F_{I, \alpha\left(R, \sigma_{i j}\right)}$ are the acting forces and $K_{k, \alpha\left(R, C_{i j k l}\right)}$ is the stiffness of the configuration.

As for a given granular medium, the energy depends just on the stresses and on the arrangement, the phase space is defined by these parameters. Therefore the partition function (6) can be rewritten as:

$$
Z_{1(\beta)}=\sum_{\alpha} \int_{\Gamma_{\alpha}} e^{-\frac{1}{2} \sum_{I=1}^{N \alpha} \frac{F_{I, \alpha\left(R, \sigma_{i j}\right)}^{2}}{K_{k, \alpha\left(R, C_{i j k l}\right)}} \beta} \mathrm{d} \sigma_{i j}
$$


$\Gamma_{\alpha}$ is the region of the complete phase space in which every configuration is possible.

Using equation (8) it is possible to obtain the value of any variable depending on the stresses or on the configuration. In particular the packing ratio $\Phi_{\alpha(\sigma i j)}$, which is a function of the configuration and, sometimes, is also function of the stresses. The expression is:

$$
\frac{1}{<\Phi>}=\frac{\sum_{\alpha} \int_{\Gamma_{\alpha}} \frac{1}{\Phi_{\alpha\left(\sigma_{i j}\right)}} e^{-H_{1, \alpha\left(\sigma_{i j}\right)} \beta} \mathrm{d} \sigma_{i j}}{\sum_{\alpha} \int_{\Gamma_{\alpha}} e^{-H_{1, \alpha\left(\sigma_{i j}\right)} \beta} \mathrm{d} \sigma_{i j}} \neq f_{(\beta)}
$$

The average compacity does not depend on $\beta$. It just relates to the volume of the phase space and the stiffness of every possible configuration, which is an intrinsic property of every granular medium.

The parameter $\beta$ is equal to the inverse average elastic potential energy, i. e. it depends on quadratic functions of the stresses.

$$
\frac{1}{\beta}=\frac{1}{2} \sigma_{e x t, i j} S_{e q, i j k l} \sigma_{e x t, k l}
$$

being $S_{e q, i j k l}$ the equivalent compliance tensor of the whole medium. For the case of isotropic compression, an expression to obtain the equivalent volumetric stiffness $K_{e q}$ is given in [9].

Therefore this new approach allows to obtain the average compacity of the most probable state and other average values of a granular medium just by determining possible equilibrium configurations and by establishing the allowed part of the phase space and the relationship between the stresses and the energy of the particles. For 2D monodisperse systems (the simplest case) and just considering two Crystal-like arrangements, the expected value matches the reported values reasonably (about $\Phi \simeq 0.84-0.86$. See [9]).

\section{UNDERLYING PRINCIPLES OF GRANULAR MEDIA}

\subsection{ENERGY AND ENTROPY}

The solution of the elastic problem of a conventional continuum medium can be obtained using the techniques of calculus of variations. The potential energy is stationary if the solid is in equilibrium, and furthermore, the equilibrium is stable if the potential energy reaches a minimum (principle of minimum potential energy). This formulation is equivalent to solving the equations of elasticity.

However, if the granular medium is considered as a set of individual particles, this principle is not appropriate because the whole system does not tend to minimize the energy. In fact, the minimum potential energy state(considering both elastic and gravitational 
energies) corresponds to the most dense arrangement (a regular lattice for monodisperse media) and, intuitively, this situation is too ordered to be achieved.

Some methods used for modelling the behavior of granular media, as FEMs, use the principle of minimum potential energy but for an approximate continuum medium in which constitutive relationships intrinsically consider particles rearrangement and the consequent variation on the stress-strain behavior. Other methods, as DEMs, apply dynamic and static laws to all the particles. As not only the initial conditions but also the interaction between particles are considered, the resulting state achieved after a calculation can be realistic (and it does not correspond to the minimum potential energy state).

The establishment of the features of the equilibrium state of disordered arrangements could be useful to solve these problems in an alternative way which considers not only the internal energy but also the order. Order and probability are concepts joined by entropy. Boltzmann (1898) [7,6] proposed that the entropy relates to the number of possible microstates $\Omega$ in the way ${ }^{3}$ :

$$
S \propto \ln \Omega
$$

Boltzmann went on to show that this definition of entropy was equivalent to the thermodynamic entropy to within a constant number which has since been known as Boltzmann's constant $k_{B}$.

However the definition of entropy, which comes from Statistical Mechanics, rather than thermodynamics, is extensible to any system of an ensemble (even when the temperature is not determinant). The entropy is essentially a measure of the number of ways in which a system may be arranged and it is often taken to be a measure of "disorder" (the higher the entropy, the higher the disorder). Thus the definition of entropy given by the Gibbs entropy formula $[7,6]$ :

$$
S \propto-\sum_{i} P_{i} \ln P_{i}
$$

This definition remains valid even when the system is far away from equilibrium. Moreover the $2^{\text {nd }}$ Law of Thermodynamics states that in general the total entropy of any system will not decrease other than by increasing the entropy of some other system, so that the maximization of this quantity states the equilibrium condition.

The observations of disordered granular media evidence that the features of the arrangement are universal and repeatable (if the protocols are the same). This has conducted to the consideration of a kind of entropy of granular media together with the energy state of particles. The equilibrium of disordered granular systems does not coincide with the state of minimum potential energy but with the state of maximum entropy. And overcoming

\footnotetext{
${ }^{3}$ The reason is that entropy is an additive property while probability is multiplicative
} 
this situation would require the introduction of order to the system, what is less probable. Thus, entropy explains that there is a well-established limit to the achievable density.

This approach could start a new way of solving problems, via describing the most probable state through the maximization of the entropy (14). However, it is important to remark that this minimization gives the state of equilibrium, which is not immediately attained with just a modification of the conditions of the problem. Knowing which is the actual behavior of a granular material after a modification of the conditions is not still possible and to do that a theory of evolution to the equilibrium is necessary.

\subsection{THE PROBABILITY OF EVERY ARRANGEMENT AT EQUILIB- RIUM}

Both approaches included in sections 3.2 and 3.3, show that not all the arrangements are equally likely to be present.

The Edward's approach states a distribution function in which the probability of every arrangement depends on the relative volume and on a new property of granular media, the frothiness (which scales the distribution). The new proposal states that the probability of every configuration depends on the volume of the phase space allowed, and on quadratic forms of stresses.

In the equilibrium state, the distribution function of the arrangement is expected to be always the same so this is an underlying principle which had to be satisfied. On the other hand, statistical mechanics approaches could be useful for the establishment of some patterns of assemblies in equilibrium.

This considerations could be helpful for the first step in discrete element simulations: the generation of the initial assembly. Nevertheless, preparing an initial distribution of a large number of particles in a realistic and random-like manner is not trivial and it concerns to a lot of researchers.

Two kinds of methods for the preparation of dense arrangements for simulations are possible (for a review, see [10]): dynamic methods (or non-constructive) and constructive algorithms. Dynamic methods use the DEM code itself for the preparation of the sample, by different ways: increasing diameters, moving slowly the walls of the domain (and allowing particles to be rearranged) or by gravitational deposition; constructive methods are less time-consuming and their basic feature is that assemblies are prepared with the help of purely geometrical calculations, without simulating the dynamics of particle motion.

Additional techniques to generate realistic initial samples, as well as the need to develop appropriate algorithms have been highlighted by some researchers $[11,12]$ However, it is not definitely well-established what is a good sample. Probably the distinction depends on the desired precision. Apparently, engineers are more concerned about the time consumed to obtain a dense arrangement (within a precision range of about $1 \%$ ). On the other hand, physicists are concerned about the definition of the ground state of granular 
media (arising concepts as random close and random loose packing).

Starting from the hypothesis that identical granular media (i.e. those described by the same particle shape and size distribution, friction factor, and other parameters) give the same response to the same protocol of arrangement, statistical mechanics approaches try to define the macroscopic features which the system attains. These approaches differentiate equilibrium states (described by an equation of state) and states out of equilibrium (which would be more or less sensible to external driving and which would tend to the equilibrium situation). In consequence, they could illuminate how likely is every generated arrangement. Moreover, if those arrangements corresponding to the equilibrium state were defined, we could avoid the generation of initial assemblies in every simulation.

\subsection{THE MOST PROBABLE AVERAGE PACKING RATIO}

Once the distribution function of the phase space is known, the average value of variables which depend on the phase space coordinates can be computed using (8). According to the approach mentioned above, the expected packing ratio at equilibrium is given by (11) and it does not depend on $\beta$. This lead us to the conclusion that the expected value of the density of a granular medium is not affected by the stress level but by whether the system is in equilibrium or not. Therefore $\Phi$ is an intrinsic property of the medium (which depends on the shape, size-distribution and friction of particles). Although granular media can be found in looser or denser configurations, driving the system (by tapping or cyclic compression, for instance) would make it to tend towards the equilibrium state. However, once this situation has been reached, the same process does not make it to abandon that state (that would require the introduction of order into the system). This reasoning can be helpful to explain some concepts, such as random close packing and evidences why granular systems always tend to attain close values of packing ratio. Nevertheless, some experimental results have shown that the attained average packing ratio depends on the protocol used to configure the arrangement. This is probably due to the fact that the equilibrium state is not always totally reached. Although this is still an open question, the relative differences among reached states are mostly within the desired precision for engineering purposes.

If the equilibrium state of a granular medium is determined, the variations of the average packing ratio (and other properties) could be determined by the analysis of the evolution from initial (out of equilibrium) states to the equilibrium state. Nevertheless a proper framework has to be developed.

\section{CONCLUSION}

Statistical Mechanics could be an useful tool to explain certain aspects of the behavior of granular materials. 
First results have shown that these analytical particle-based methods could complement other numerical particle-based techniques (such as DEM or Molecular Dynamics) or continuum models (used in FE and Finite Difference Methods).

Consideration of these results could be useful for the generation of initial arrangements and the analysis of the evolution towards the equilibrium situation, although more research is still needed to establish a complete framework for the analysis of several media. In such research numerical results and theoretical developments should be performed at the same time.

\section{REFERENCES}

[1] Lade, P. V. Overview of constitutive models for soils, ASCE Conf. Proc. (2005), 165 (1): $1-34$.

[2] Soga, K. and O'Sullivan C. Modeling of geomaterials behavior. Soils and Foundations(2010), 50 (6):861-875.

[3] Fukue, M., and Mulligan, C. M. Equations of state in soil compression based on Statistical Mechanics. Soils and Foundations, (2009), 49 (1): 99-114.

[4] Tejada, I. G. and Jimenez, R. Equations of state in soil compression based on Statistical Mechanics (discussion). Soils and Foundations, (2010), 50 (2): 335-336.

[5] Cundall, P. and Strack, O. A discrete numerical model for granular assemblies. Geotechnique, (1979), 29 (1): 47-65.

[6] Balescu, R. Equilibrium and nonequilibrium statistical mechanics, John Willey \& Sons, (1975).

[7] Pathria, R. K. Statistical mechanics. Butterworth-Heinemann (Elsevier), (1972).

[8] Edwards, S. and Oakeshott, R. Theory of powders. Physica A, (1989), 157 (3):10801090.

[9] Tejada, I. G. A new statistical mechanics approach to dense granular media. Physica A (2011) 390 (14): 2664-2677.

[10] Bagi, K. An algorithm to generate random dense arrangements for discrete element simulations of granular assemblies. Granular Matter, (2005), 7: 31-43.

[11] O' Sullivan, C. The Application of Discrete Element Modelling to Finite Deformation Problems in Geomechanics. Ph. D. thesis. University of California, Berkeley, (2002).

[12] Feng, Y. T., Han, K. and Owen, D. R. J. Filling domains with disks: An advancing front approach, Inter. J. Numer. Methods in Engineering, (2003), 56 (5): 699-731. 